\title{
New Existence Results for Nonlinear Fractional Differential Equations with Three-Point Integral Boundary Conditions
}

\author{
Bashir Ahmad, ${ }^{1}$ Sotiris K. Ntouyas, ${ }^{2}$ and Ahmed Alsaedi ${ }^{1}$ \\ ${ }^{1}$ Department of Mathematics, Faculty of Science, King Abdulaziz University, P.O. Box 80203, \\ Jeddah 21589, Saudi Arabia \\ ${ }^{2}$ Department of Mathematics, University of Ioannina, 45110 Ioannina, Greece
}

Correspondence should be addressed to Bashir Ahmad, bashir_qau@yahoo.com

Received 30 October 2010; Revised 12 December 2010; Accepted 12 December 2010

Academic Editor: Dumitru Baleanu

Copyright (C) 2011 Bashir Ahmad et al. This is an open access article distributed under the Creative Commons Attribution License, which permits unrestricted use, distribution, and reproduction in any medium, provided the original work is properly cited.

\begin{abstract}
This paper studies a boundary value problem of nonlinear fractional differential equations of order $q \in(1,2]$ with three-point integral boundary conditions. Some new existence and uniqueness results are obtained by using standard fixed point theorems and Leray-Schauder degree theory. Our results are new in the sense that the nonlocal parameter in three-point integral boundary conditions appears in the integral part of the conditions in contrast to the available literature on three-point boundary value problems which deals with the three-point boundary conditions restrictions on the solution or gradient of the solution of the problem. Some illustrative examples are also discussed.
\end{abstract}

\section{Introduction}

In recent years, boundary value problems for nonlinear fractional differential equations have been addressed by several researchers. Fractional derivatives provide an excellent tool for the description of memory and hereditary properties of various materials and processes; see [1]. These characteristics of the fractional derivatives make the fractionalorder models more realistic and practical than the classical integer-order models. As a matter of fact, fractional differential equations arise in many engineering and scientific disciplines such as physics, chemistry, biology, economics, control theory, signal and image processing, biophysics, blood flow phenomena, aerodynamics, and fitting of experimental data, [1-4]. For some recent development on the topic, see [5-21] and the references therein. 
We discuss the existence and uniqueness of solutions for a boundary value problem of nonlinear fractional differential equations of order $q \in(1,2]$ with three-point integral boundary conditions given by

$$
\begin{aligned}
& { }^{c} D^{q} x(t)=f(t, x(t)), \quad 0<t<1,1<q \leq 2, \\
& x(0)=0, \quad x(1)=\alpha \int_{0}^{\eta} x(s) d s, \quad 0<\eta<1,
\end{aligned}
$$

where ${ }^{c} D^{q}$ denotes the Caputo fractional derivative of order $q, f:[0,1] \times X \rightarrow X$ is continuous, and $\alpha \in \mathbb{R}$ is such that $\alpha \neq 2 / \eta^{2}$. Here, $(X,\|\cdot\|)$ is a Banach space and $C=$ $C([0,1], X)$ denotes the Banach space of all continuous functions from $[0,1] \rightarrow X$ endowed with a topology of uniform convergence with the norm denoted by $\|\cdot\|$.

Note that the three-point boundary condition in (1.1) corresponds to the area under the curve of solutions $x(t)$ from $t=0$ to $t=\eta$.

\section{Preliminaries}

Let us recall some basic definitions of fractional calculus $[2,4]$.

Definition 2.1. For a continuous function $g:[0, \infty) \rightarrow \mathbb{R}$, the Caputo derivative of fractional order $q$ is defined as

$$
{ }^{c} D^{q} g(t)=\frac{1}{\Gamma(n-q)} \int_{0}^{t}(t-s)^{n-q-1} g^{(n)}(s) d s, \quad n-1<q<n, n=[q]+1,
$$

where $[q]$ denotes the integer part of the real number $q$.

Definition 2.2. The Riemann-Liouville fractional integral of order $q$ is defined as

$$
I^{q} g(t)=\frac{1}{\Gamma(q)} \int_{0}^{t} \frac{g(s)}{(t-s)^{1-q}} d s, \quad q>0
$$

provided the integral exists.

Definition 2.3. The Riemann-Liouville fractional derivative of order $q$ for a continuous function $g(t)$ is defined by

$$
D^{q} g(t)=\frac{1}{\Gamma(n-q)}\left(\frac{d}{d t}\right)^{n} \int_{0}^{t} \frac{g(s)}{(t-s)^{q-n+1}} d s, \quad n=[q]+1,
$$

provided the right-hand side is pointwise defined on $(0, \infty)$. 
Lemma 2.4 (see [2]). For $q>0$, the general solution of the fractional differential equation ${ }^{c} D^{q} x(t)=$ 0 is given by

$$
x(t)=c_{0}+c_{1} t+c_{2} t^{2}+\cdots+c_{n-1} t^{n-1},
$$

where $c_{i} \in \mathbb{R}, i=0,1,2, \ldots, n-1(n=[q]+1)$.

In view of Lemma 2.4, it follows that

$$
I^{q c} D^{q} x(t)=x(t)+c_{0}+c_{1} t+c_{2} t^{2}+\cdots+c_{n-1} t^{n-1},
$$

for some $c_{i} \in \mathbb{R}, i=0,1,2, \ldots, n-1(n=[q]+1)$.

Lemma 2.5. A unique solution of the boundary value problem (1.1) is given by

$$
\begin{aligned}
x(t)= & \frac{1}{\Gamma(q)} \int_{0}^{t}(t-s)^{q-1} f(s, x(s)) d s \\
& -\frac{2 t}{\left(2-\alpha \eta^{2}\right) \Gamma(q)} \int_{0}^{1}(1-s)^{q-1} f(s, x(s)) d s \\
& +\frac{2 \alpha t}{\left(2-\alpha \eta^{2}\right) \Gamma(q)} \int_{0}^{\eta}\left(\int_{0}^{s}(s-m)^{q-1} f(m, x(m)) d m\right) d s .
\end{aligned}
$$

Proof. For some constants $c_{0}, c_{1} \in X$, we have

$$
x(t)=I^{q} \rho(t)-c_{0}-c_{1} t=\int_{0}^{t} \frac{(t-s)^{q-1}}{\Gamma(q)} y(s) d s-c_{0}-c_{1} t
$$

From $x(0)=0$, we have $c_{0}=0$. Applying the second boundary condition for (1.1), we find that

$$
\begin{aligned}
\alpha \int_{0}^{\eta} x(s) d s= & \alpha \int_{0}^{\eta}\left(\int_{0}^{s} \frac{(s-m)^{q-1}}{\Gamma(q)} f(m, x(m)) d m-c_{1} s\right) d s \\
= & \alpha \int_{0}^{\eta}\left(\int_{0}^{s} \frac{(s-m)^{q-1}}{\Gamma(q)} f(m, x(m)) d m\right) d s-\alpha c_{1} \frac{\eta^{2}}{2} \\
& x(1)=\int_{0}^{1} \frac{(1-s)^{q-1}}{\Gamma(q)} f(s, x(s)) d s-c_{1},
\end{aligned}
$$


which imply that

$$
c_{1}=\frac{2}{2-\alpha \eta^{2}}\left[\int_{0}^{1} \frac{(1-s)^{q-1}}{\Gamma(q)} f(s, x(s)) d s-\alpha \int_{0}^{\eta}\left(\int_{0}^{s} \frac{(s-m)^{q-1}}{\Gamma(q)} f(m, x(m)) d m\right) d s\right] .
$$

Substituting the values of $c_{0}$ and $c_{1}$ in (2.7), we obtain the solution (2.6).

In view of Lemma 2.5 , we define an operator $\mathbf{F}: \mathcal{C} \rightarrow \mathcal{C}$ by

$$
\begin{aligned}
(\mathbf{F} x)(t)= & \frac{1}{\Gamma(q)} \int_{0}^{t}(t-s)^{q-1} f(s, x(s)) d s \\
& -\frac{2 t}{\left(2-\alpha \eta^{2}\right) \Gamma(q)} \int_{0}^{1}(1-s)^{q-1} f(s, x(s)) d s \\
& +\frac{2 \alpha t}{\left(2-\alpha \eta^{2}\right) \Gamma(q)} \int_{0}^{\eta}\left(\int_{0}^{s}(s-m)^{q-1} f(m, x(m)) d m\right) d s, \quad t \in[0,1] .
\end{aligned}
$$

To prove the main results, we need the following assumptions:

$\left(\mathrm{A}_{1}\right)\|f(t, x)-f(t, y)\| \leq L\|x-y\|$, for all $t \in[0,1], L>0, x, y \in X$;

$\left(\mathrm{A}_{2}\right)\|f(t, x)\| \leq \mu(t)$, for all $(t, x) \in[0,1] \times X$, and $\mu \in L^{1}\left([0,1], R^{+}\right)$.

For convenience, let us set

$$
\Lambda=\frac{1}{\Gamma(q+1)}\left(1+\frac{2\left[(q+1)+|\alpha| \eta^{q+1}\right]}{\left|2-\alpha \eta^{2}\right|(q+1)}\right)
$$

\section{Existence Results in a Banach Space}

Theorem 3.1. Assume that $f:[0,1] \times X \rightarrow X$ is a jointly continuous function and satisfies the assumption $\left(A_{1}\right)$ with $L<1 / \Lambda$, where $\Lambda$ is given by (2.11). Then the boundary value problem (1.1) has a unique solution. 
Proof. Setting $\sup _{t \in[0,1]}|f(t, 0)|=M$ and choosing $r \geq \Lambda M /(1-L \Lambda)$, we show that $\mathbf{F} B_{r} \subset B_{r}$, where $B_{r}=\{x \in \mathcal{C}:\|x\| \leq r\}$. For $x \in B_{r}$, we have

$$
\begin{aligned}
& \|(\mathbf{F} x)(t)\| \leq \frac{1}{\Gamma(q)} \int_{0}^{t}(t-s)^{q-1}\|f(s, x(s))\| d s \\
& +\left|\frac{2 t}{\left(2-\alpha \eta^{2}\right) \Gamma(q)}\right| \int_{0}^{1}(1-s)^{q-1}\|f(s, x(s))\| d s \\
& +\left|\frac{2 \alpha t}{\left(2-\alpha \eta^{2}\right) \Gamma(q)}\right| \int_{0}^{\eta}\left(\int_{0}^{s}(s-m)^{q-1}\|f(m, x(m))\| d m\right) d s \\
& \leq \frac{1}{\Gamma(q)} \int_{0}^{t}(t-s)^{q-1}(\|f(s, x(s))-f(s, 0)\|+\|f(s, 0)\|) d s \\
& +\left|\frac{2 t}{\left(2-\alpha \eta^{2}\right) \Gamma(q)}\right| \int_{0}^{1}(1-s)^{q-1}(\|f(s, x(s))-f(s, 0)\|+\|f(s, 0)\|) d s \\
& +\left|\frac{2 \alpha t}{\left(2-\alpha \eta^{2}\right) \Gamma(q)}\right| \int_{0}^{\eta}\left(\int_{0}^{s}(s-m)^{q-1}(\|f(m, x(m))-f(m, 0)\|+\|f(m, 0)\|) d m\right) d s \\
& \leq(L r+M)\left[\frac{1}{\Gamma(q)} \int_{0}^{t}(t-s)^{q-1} d s+\left|\frac{2 t}{\left(2-\alpha \eta^{2}\right) \Gamma(q)}\right| \int_{0}^{1}(1-s)^{q-1} d s\right. \\
& \left.+\left|\frac{2 \alpha t}{\left(2-\alpha \eta^{2}\right) \Gamma(q)}\right| \int_{0}^{\eta}\left(\int_{0}^{s}(s-m)^{q-1} d m\right) d s\right] \\
& \leq \frac{(L r+M)}{\Gamma(q+1)}\left(1+\frac{2\left[(q+1)+|\alpha| \eta^{q+1}\right]}{\left|2-\alpha \eta^{2}\right|(q+1)}\right) \\
& =(L r+M) \Lambda \leq r .
\end{aligned}
$$

Now, for $x, y \in \mathcal{C}$ and for each $t \in[0,1]$, we obtain

$$
\begin{aligned}
\|(\mathbf{F} x)(t)-(\mathbf{F} y)(t)\| \leq & \frac{1}{\Gamma(q)} \int_{0}^{t}(t-s)^{q-1}\|f(s, x(s))-f(s, y(s))\| d s \\
& +\left|\frac{2 t}{\left(2-\alpha \eta^{2}\right) \Gamma(q)}\right| \int_{0}^{1}(1-s)^{q-1}\|f(s, x(s))-f(s, y(s))\| d s \\
& +\left|\frac{2 \alpha t}{\left(2-\alpha \eta^{2}\right) \Gamma(q)}\right| \int_{0}^{\eta}\left(\int_{0}^{s}(s-m)^{q-1}\|f(m, x(m))-f(m, y(m))\| d m\right) d s
\end{aligned}
$$




$$
\begin{gathered}
\leq L\|x-y\|\left[\frac{1}{\Gamma(q)} \int_{0}^{t}(t-s)^{q-1} d s+\left|\frac{2 t}{\left(2-\alpha \eta^{2}\right) \Gamma(q)}\right| \int_{0}^{1}(1-s)^{q-1} d s\right. \\
\left.+\left|\frac{2 \alpha t}{\left(2-\alpha \eta^{2}\right) \Gamma(q)}\right| \int_{0}^{\eta}\left(\int_{0}^{s}(s-m)^{q-1} d m\right) d s\right] \\
\leq \frac{L}{\Gamma(q+1)}\left(1+\frac{2\left[(q+1)+|\alpha| \eta^{q+1}\right]}{\left|2-\alpha \eta^{2}\right|(q+1)}\right)\|x-y\|=L \Lambda\|x-y\|,
\end{gathered}
$$

where $\Lambda$ is given by (2.11). Observe that $\Lambda$ depends only on the parameters involved in the problem. As $L<1 / \Lambda$, therefore $\mathbf{F}$ is a contraction. Thus, the conclusion of the theorem follows by the contraction mapping principle (Banach fixed point theorem).

Now, we prove the existence of solutions of (1.1) by applying Krasnoselskii's fixed point theorem [22].

Theorem 3.2 (Krasnoselskii's fixed point theorem). Let $M$ be a closed convex and nonempty subset of a Banach space $X$. Let $A, B$ be the operators such that (i) $A x+B y \in M$ whenever $x, y \in M$; (ii) $A$ is compact and continuous; (iii) $B$ is a contraction mapping. Then there exists $z \in M$ such that $z=A z+B z$.

Theorem 3.3. Let $f:[0,1] \times X \rightarrow X$ be a jointly continuous function mapping bounded subsets of $[0,1] \times X$ into relatively compact subsets of $X$, and the assumptions $\left(A_{1}\right)$ and $\left(A_{2}\right)$ hold with

$$
\frac{L}{\Gamma(q+1)}\left(\frac{2\left[(q+1)+|\alpha| \eta^{q+1}\right]}{\left|2-\alpha \eta^{2}\right|(q+1)}\right)<1
$$

Then the boundary value problem (1.1) has at least one solution on $[0,1]$.

Proof. Letting $\sup _{t \in[0,1]}|\mu(t)|=\|\mu\|$, we fix

$$
\bar{r} \geq \frac{\|\mu\|}{\Gamma(q+1)}\left(1+\frac{2\left[(q+1)+|\alpha| \eta^{q+1}\right]}{\left|2-\alpha \eta^{2}\right|(q+1)}\right)
$$

and consider $B_{\bar{r}}=\{x \in \mathcal{C}:\|x\| \leq \bar{r}\}$. We define the operators $D$ and $\mathcal{Q}$ on $B_{\bar{r}}$ as

$$
\begin{aligned}
(D x)(t)= & \int_{0}^{t} \frac{(t-s)^{q-1}}{\Gamma(q)} f(s, u(s)) d s \\
(Q x)(t)= & -\frac{2 t}{\left(2-\alpha \eta^{2}\right) \Gamma(q)} \int_{0}^{1}(1-s)^{q-1} f(s, x(s)) d s \\
& +\frac{2 \alpha t}{\left(2-\alpha \eta^{2}\right) \Gamma(q)} \int_{0}^{\eta}\left(\int_{0}^{s}(s-m)^{q-1} f(m, x(m)) d m\right) d s .
\end{aligned}
$$


For $x, y \in B_{\bar{r}}$, we find that

$$
\|P x+Q y\| \leq \frac{\|\mu\|}{\Gamma(q+1)}\left(1+\frac{2\left[(q+1)+|\alpha| \eta^{q+1}\right]}{\left|2-\alpha \eta^{2}\right|(q+1)}\right) \leq \bar{r} .
$$

Thus, $p x+Q y \in B_{\bar{r}}$. It follows from the assumption $\left(A_{1}\right)$ together with (3.3) that $Q$ is a contraction mapping. Continuity of $f$ implies that the operator $D$ is continuous. Also, $D$ is uniformly bounded on $B_{\bar{r}}$ as

$$
\|p x\| \leq \frac{\|\mu\|}{\Gamma(q+1)}
$$

Now we prove the compactness of the operator $p$.

In view of $\left(A_{1}\right)$, we define $\sup _{(t, x) \in[0,1] \times B_{\bar{r}}}|f(t, x)|=\bar{f}$, and consequently we have

$$
\begin{aligned}
& \left\|(D x)\left(t_{1}\right)-(D x)\left(t_{2}\right)\right\| \\
& =\left\|\frac{1}{\Gamma(q)} \int_{0}^{t_{1}}\left[\left(t_{2}-s\right)^{q-1}-\left(t_{1}-s\right)^{q-1}\right] f(s, x(s)) d s+\int_{t_{1}}^{t_{2}}\left(t_{2}-s\right)^{q-1} f(s, x(s)) d s\right\| \\
& \leq \frac{\bar{f}}{\Gamma(q+1)}\left|2\left(t_{2}-t_{1}\right)^{q}+t_{1}^{q}-t_{2}^{q}\right|
\end{aligned}
$$

which is independent of $x$. Thus, $D$ is equicontinuous. Using the fact that $f$ maps bounded subsets into relatively compact subsets, we have that $D(\mathscr{A})(t)$ is relatively compact in $X$ for every $t$, where $A$ is a bounded subset of $\mathcal{C}$. So $D$ is relatively compact on $B_{\bar{r}}$. Hence, by the Arzelá-Ascoli Theorem, $D$ is compact on $B_{\bar{r}}$. Thus all the assumptions of Theorem 3.2 are satisfied. So the conclusion of Theorem 3.2 implies that the boundary value problem (1.1) has at least one solution on $[0,1]$.

\section{Existence of Solution via Leray-Schauder Degree Theory}

Theorem 4.1. Let $f:[0,1] \times \mathbb{R} \rightarrow \mathbb{R}$. Assume that there exist constants $0 \leq \kappa<1 / \Lambda$, where $\Lambda$ is given by (2.11) and $M>0$ such that $|f(t, x)| \leq \kappa|x|+M$ for all $t \in[0,1], x \in C[0,1]$. Then the boundary value problem (1.1) has at least one solution.

Proof. Let us define an operator $\digamma: C[0,1] \rightarrow C[0,1]$ as

$$
x=\digamma x,
$$


where

$$
\begin{aligned}
(\digamma x)(t)= & \frac{1}{\Gamma(q)} \int_{0}^{t}(t-s)^{q-1} f(s, x(s)) d s \\
& -\frac{2 t}{\left(2-\alpha \eta^{2}\right) \Gamma(q)} \int_{0}^{1}(1-s)^{q-1} f(s, x(s)) d s \\
& +\frac{2 \alpha t}{\left(2-\alpha \eta^{2}\right) \Gamma(q)} \int_{0}^{\eta}\left(\int_{0}^{s}(s-m)^{q-1} f(m, x(m)) d m\right) d s .
\end{aligned}
$$

In view of the fixed point problem (4.1), we just need to prove the existence of at least one solution $x \in C[0,1]$ satisfying (4.1). Define a suitable ball $B_{R} \subset C[0,1]$ with radius $R>0$ as

$$
B_{R}=\left\{x \in C[0,1]: \max _{t \in[0,1]}|x(t)|<R\right\}
$$

where $R$ will be fixed later. Then, it is sufficient to show that $\digamma: \bar{B}_{R} \rightarrow C[0,1]$ satisfies

$$
x \neq \lambda \digamma x, \quad \forall x \in \partial B_{R}, \forall \lambda \in[0,1] .
$$

Let us set

$$
H(\lambda, x)=\lambda \digamma x, \quad x \in C(\mathbb{R}), \lambda \in[0,1] .
$$

Then, by the Arzelá-Ascoli Theorem, $h_{\lambda}(x)=x-H(\lambda, x)=x-\lambda \digamma x$ is completely continuous. If (4.4) is true, then the following Leray-Schauder degrees are well defined and by the homotopy invariance of topological degree, it follows that

$$
\begin{aligned}
\operatorname{deg}\left(h_{\lambda}, B_{R}, 0\right) & =\operatorname{deg}\left(I-\lambda \digamma, B_{R}, 0\right)=\operatorname{deg}\left(h_{1}, B_{R}, 0\right) \\
& =\operatorname{deg}\left(h_{0}, B_{R}, 0\right)=\operatorname{deg}\left(I, B_{R}, 0\right)=1 \neq 0, \quad 0 \in B_{r},
\end{aligned}
$$

where $I$ denotes the unit operator. By the nonzero property of Leray-Schauder degree, $h_{1}(t)=$ $x-\lambda \digamma x=0$ for at least one $x \in B_{R}$. In order to prove (4.4), we assume that $x=\lambda \digamma x$ for some 
$\lambda \in[0,1]$ and for all $t \in[0,1]$ so that

$$
\begin{aligned}
|x(t)|= & |\lambda(\digamma x)(t)| \\
\leq & \frac{1}{\Gamma(q)} \int_{0}^{t}(t-s)^{q-1}\|f(s, x(s))\| d s \\
& +\left|\frac{2 t}{\left(2-\alpha \eta^{2}\right) \Gamma(q)}\right| \int_{0}^{1}(1-s)^{q-1}\|f(s, x(s))\| d s \\
& +\left|\frac{2 \alpha t}{\left(2-\alpha \eta^{2}\right) \Gamma(q)}\right| \int_{0}^{\eta}\left(\int_{0}^{s}(s-m)^{q-1}\|f(m, x(m))\| d m\right) d s \\
\leq & (\kappa|x|+M)\left[\frac{1}{\Gamma(q)} \int_{0}^{t}(t-s)^{q-1} d s+\left|\frac{2 t}{\left(2-\alpha \eta^{2}\right) \Gamma(q)}\right| \int_{0}^{1}(1-s)^{q-1} d s\right. \\
\leq & \frac{\kappa|x|+M}{\Gamma(q+1)}\left(1+\frac{2\left[(q+1)+|\alpha| \eta^{q+1}\right]}{\left|2-\alpha \eta^{2}\right|(q+1)}\right) \\
= & (\kappa|x|+M) \Lambda, \\
& \left.+\frac{2 \alpha t}{\left(2-\alpha \eta^{2}\right) \Gamma(q)} \mid \int_{0}^{\eta}\left(\int_{0}^{s}(s-m)^{q-1} d m\right) d s\right]
\end{aligned}
$$

which, on taking norm $\left(\sup _{t \in[0,1]}|x(t)|=\|x\|\right)$ and solving for $\|x\|$, yields

$$
\|x\| \leq \frac{M \Lambda}{1-\kappa \Lambda}
$$

Letting $R=M \Lambda /(1-\kappa \Lambda)+1,(4.4)$ holds. This completes the proof.

\section{Examples}

Example 5.1. Consider the following three-point integral fractional boundary value problem:

$$
\begin{gathered}
{ }^{c} D^{3 / 2} x(t)=\frac{1}{(t+9)^{2}} \frac{\|x\|}{1+\|x\|}, \quad t \in[0,1], \\
x(0)=0, \quad x(1)=\int_{0}^{3 / 4} x(s) d s .
\end{gathered}
$$


Here, $q=3 / 2, \alpha=1, \eta=3 / 4$, and $f(t, x)=\left(1 /(t+9)^{2}\right)(\|x\| /(1+\|x\|))$. As $\| f(t, x)-$ $f(t, y)\|\leq(1 / 81)\| x-y \|$, therefore, $\left(\mathrm{A}_{1}\right)$ is satisfied with $L_{1}=1 / 81$. Further,

$$
L \Lambda=L \Lambda=\frac{L}{\Gamma(q+1)}\left(1+\frac{2\left[(q+1)+|\alpha| \eta^{q+1}\right]}{\left|2-\alpha \eta^{2}\right|(q+1)}\right)=\frac{4}{27945 \sqrt{\pi}}(275+18 \sqrt{3})<1
$$

Thus, by the conclusion of Theorem 3.1, the boundary value problem (5.1) has a unique solution on $[0,1]$.

Example 5.2. Consider the following boundary value problem:

$$
\begin{gathered}
{ }^{c} D^{3 / 2} x(t)=\frac{1}{(4 \pi)} \sin (2 \pi x)+\frac{|x|}{1+|x|}, \quad t \in[0,1], 1<q \leq 2, \\
x(0)=0, \quad x(1)=\int_{0}^{1 / 2} x(s) d s .
\end{gathered}
$$

Here, $q=3 / 2, \alpha=1, \eta=1 / 2$, and

$$
|f(t, x)|=\left|\frac{1}{(4 \pi)} \sin (2 \pi x)+\frac{|x|}{1+|x|}\right| \leq \frac{1}{2}|x|+1 .
$$

Clearly $M=1$ and

$$
\kappa=\frac{1}{2}<\frac{1}{\Lambda}=\frac{105 \sqrt{2 \pi}}{4(75 \sqrt{2}+4)}=0.5978138748
$$

Thus, all the conditions of Theorem 4.1 are satisfied and consequently the problem (5.3) has at least one solution.

\section{References}

[1] I . Podlubny, Fractional Differential Equations, vol. 198 of Mathematics in Science and Engineering, Academic Press, San Diego, Calif, USA, 1999.

[2] A. A. Kilbas, H. M. Srivastava, and J. J. Trujillo, Theory and Applications of Fractional Differential Equations, vol. 204 of North-Holland Mathematics Studies, Elsevier, Amsterdam, The Netherlands, 2006.

[3] J. Sabatier, O. P. Agrawal, and J. A. T. Machado, Eds., Advances in Fractional Calculus: Theoretical Developments and Applications in Physics and Engineering, Springer, Dordrecht, The Netherlands, 2007.

[4] S. G. Samko, A. A. Kilbas, and O. I. Marichev, Fractional Integrals and Derivatives, Theory and Applications, Gordon and Breach Science, Yverdon, Switzerland, 1993.

[5] R. P. Agarwal, B. de Andrade, and C. Cuevas, "Weighted pseudo-almost periodic solutions of a class of semilinear fractional differential equations," Nonlinear Analysis: Real World Applications, vol. 11, no. 5, pp. 3532-3554, 2010.

[6] R. P. Agarwal, V. Lakshmikantham, and J. J. Nieto, "On the concept of solution for fractional differential equations with uncertainty," Nonlinear Analysis: Theory, Methods \& Applications, vol. 72, no. 6, pp. 2859-2862, 2010.

[7] R. P. Agarwal, Y. Zhou, and Y. He, "Existence of fractional neutral functional differential equations," Computers E Mathematics with Applications, vol. 59, no. 3, pp. 1095-1100, 2010. 
[8] B. Ahmad and S. Sivasundaram, "On four-point nonlocal boundary value problems of nonlinear integro-differential equations of fractional order," Applied Mathematics and Computation, vol. 217, no. 2, pp. 480-487, 2010.

[9] B. Ahmad, "Existence of solutions for irregular boundary value problems of nonlinear fractional differential equations," Applied Mathematics Letters, vol. 23, no. 4, pp. 390-394, 2010.

[10] B. Ahmad, "Existence of solutions for fractional differential equations of order $q \in(2,3]$ with antiperiodic boundary conditions," Journal of Applied Mathematics and Computing, vol. 34, no. 1-2, pp. 385-391, 2010.

[11] B. Ahmad and J. J. Nieto, "Existence of solutions for nonlocal boundary value problems of higherorder nonlinear fractional differential equations," Abstract and Applied Analysis, vol. 2009, Article ID 494720, 9 pages, 2009.

[12] B. Ahmad and A. Alsaedi, "Existence and uniqueness of solutions for coupled systems of higher-order nonlinear fractional differential equations," Fixed Point Theory and Applications, vol. 2010, Article ID 364560, 17 pages, 2010.

[13] B. Ahmad and J. J. Nieto, "Existence results for nonlinear boundary value problems of fractional integrodifferential equations with integral boundary conditions," Boundary Value Problems, vol. 2009, Article ID 708576, 11 pages, 2009.

[14] B. Ahmad and J. J. Nieto, "Existence results for a coupled system of nonlinear fractional differential equations with three-point boundary conditions," Computers $\mathcal{E}$ Mathematics with Applications, vol. 58, no. 9 , pp. 1838-1843, 2009.

[15] Z. Bai, "On positive solutions of a nonlocal fractional boundary value problem," Nonlinear Analysis: Theory, Methods $\mathcal{E}$ Applications, vol. 72, no. 2, pp. 916-924, 2010.

[16] K. Balachandran and J. J. Trujillo, "The nonlocal Cauchy problem for nonlinear fractional integrodifferential equations in Banach spaces," Nonlinear Analysis: Theory, Methods E Applications, vol. 72, no. 12, pp. 4587-4593, 2010.

[17] M. Benchohra, S. Hamani, and S. K. Ntouyas, "Boundary value problems for differential equations with fractional order and nonlocal conditions," Nonlinear Analysis: Theory, Methods E Applications, vol. 71, no. 7-8, pp. 2391-2396, 2009.

[18] M. P. Lazarević and A. M. Spasić, "Finite-time stability analysis of fractional order time-delay systems: Gronwall's approach," Mathematical and Computer Modelling, vol. 49, no. 3-4, pp. 475-481, 2009.

[19] J. J. Nieto, "Maximum principles for fractional differential equations derived from Mittag-Leffler functions," Applied Mathematics Letters, vol. 23, no. 10, pp. 1248-1251, 2010.

[20] Z. Wei, Q. Li, and J. Che, "Initial value problems for fractional differential equations involving Riemann-Liouville sequential fractional derivative," Journal of Mathematical Analysis and Applications, vol. 367, no. 1, pp. 260-272, 2010.

[21] S. Zhang, "Positive solutions to singular boundary value problem for nonlinear fractional differential equation," Computers \& Mathematics with Applications, vol. 59, no. 3, pp. 1300-1309, 2010.

[22] M. A. Krasnoselskii, "Two remarks on the method of successive approximations," Uspekhi Matematicheskikh Nauk, vol. 10, pp. 123-127, 1955. 